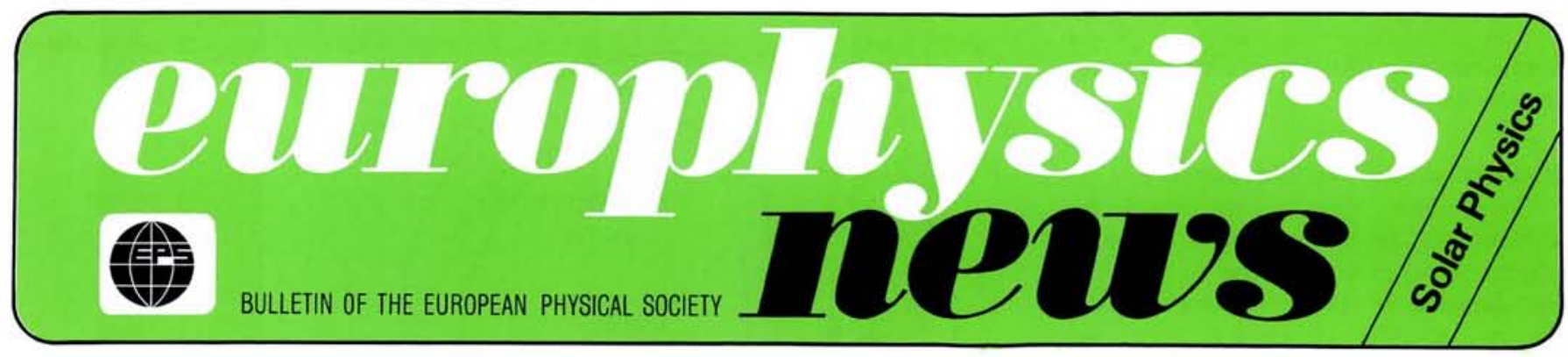

\section{Solar Physics in EPS}

On the initiative of the Board of the Solar Physics Section of the Astronomy and Astrophysics Division, this special issue of Europhysics News on solar physics has been prepared. The Solar Physics Section was established in 1976 within the EPS in order to bring physicists and astrophysicists together, and contribute to the maintenance of a greater contact across the disciplines.

Although the total number of solar physicists in Europe is substantial, the communities in most countries are small. Accordingly, the Section encourages collaboration, organizes meetings and provides a forum for the exchange of ideas. Workshops on well selected subjects have proved to be an important part of the activities and, with a view to achieving an efficient use of resources in the future, the Section organized the workshop "Near Future Plans for Solar Research" in Oxford on 10 and 11 April 1981 (see C. Jordan and I.W. Roxburgh, Europhysics News, 12, 8/9, p. 12). This workshop gave insight into the national plans of seventeen countries from all parts of Europe.

Based on the remarkable consensus that emerged concerning priorities for future study, the Section Board asked R.M. Bonnet (Verrières le Buisson), C. Jordan (Oxford) and P. Maltby (Oslo) to prepare a consolidated summary of the findings. Their report entitled "Solar Physics in Europe; recommendations for the 1980's" has recently been endorsed by the Solar Physics Section Board. In it is outlined the current state of the physical problems, and recommendations are presented for immediate action using groundbased observations, space observations, data reduction and analysis as well as theory. By giving advice to future research in this way the Solar Physics Section hopes to encourage a continued significant European contribution to the field.

\title{
Development of Solar Physics in Europe
}

\author{
Per Maltby, Oslo \\ (Institute of Theoretical Physics)
}

Chairman of the Solar Physics Section of EPS

Solar physics, indeed, the whole field of astrophysics, began in 1611 when the first observations of sunspots through a telescope were announced. The honour of this discovery was shared by: J. Goldsmid (also known as Fabricius) in Holland, G. Galilei in Italy, C. Scheiner in Germany and T. Harriot in England. The priority of publication belongs to Goldsmid, but it is clear that Galilei had noticed spots on the solar surface in July 1610 already.

\section{Development in the 19th Century}

Advances in solar physics have usually occurred following the development of new types of instrumentation or new physical concepts. For example, the sunspot observations by Galilei and others were made possible by the invention of the telescope by Lippershey and his associates in Holland. In some cases, however, results emerged from the analysis of synoptic data accumulated during a large number of years. For more than two centuries, progress in solar physics was very slow. Then in $1843, \mathrm{H}$. Schwabe, analysed his sunspot observations and found that the "spottedness" of the solar surface varied with a period of about 10 years. An accurate determination by Wolf in 1852 gave an average period of 11.1 years for the solar cycle.

As noted already by Galilei, individual sunspots have lifetimes ranging from less than one day to several months. Using observations of long lived sunspots, R.C. Carrington was able to determine quite accurately the solar rotation and its deviation from that of a rigid body in 1863 . His discovery that the average latitude of sunspots decreases steadily from the beginning to the end of each 11 year cycle, supplied the first indication of a connection between solar rotation and the occurrence of sunspots. This drift was also investigated by G. Spörer, who noted that the number of sunspots was remarkably low between 1645 and 1715 , a period that was studied in detail by E.W. Maunder in 1894 and is usually referred to as the "Maunder minimum". The phenomenon suggests that the solar cycle itself is variable, a possibility that has received considerable publicity in recent years in connection with studies of the climate of the Earth.

The next great instrumental development was the introduction of the spectroscope by Fraunhofer who, in 1814, published a description of the solar spectrum based on visual examinations. The investigation of stars by physical methods began in 1859 when Kirchhoff and Bunsen discovered the significance of the Fraunhofer lines in the solar spectrum. The discovery of helium as a new chemical element was based on observations of the $D_{3}$ line by Janssen during the eclipse of 1868. In passing, we note that it was not until 1895 that Ramsay succeeded in isolating helium from terrestrial minerals. The first detailed mapping of the solar spectrum was based on Rowland's perfection of the concave grating and was published in 1897.

The first successful photograph of the Sun was taken by Fizeau and Foucault in 1845. The nature of prominences (i.e. cold and dense regions extending up to, say, $40000 \mathrm{~km}$ or more in the solar atmosphere) was a subject of controversy until photographic records obtained by de la Rue and by Secchi during the solar eclipse in 1860 identified them as being of solar origin. It was shown by Janssen and by Lockyer in 1868 that sufficient energy is emitted by prominences for them to be observable outside the periphery of the eclipsed Sun if 